\title{
Can Older People See Something Apart from Themselves?
}

\author{
S. Pearlman-Avnion, J. N. Lazar, and T. Muschinsky
}

\begin{abstract}
Some cognitive as well as physical functions are reduced as a function of age due to aging of the body and the brain. On the other hand, older people have broad life experiences that may serve as a resilience factor to help their abilities. The literature presents two opposite kinds of findings about cognitive ability. The main purpose of the present study is to determine whether theory of mind (ToM) ability and short-term memory are reduced or enhanced across the lifespan. A second purpose is to test gender differences. A study was conducted with 73 participants, 37 females and 36 males, whose ages ranged from 50 to 92 years. Participants were presented with a Reading the Mind in the Eyes test and a free recall task. The results suggest that the older the person, the lower his or her ToM and short-term memory abilities. Males' ToM ability was found to be more impaired than that of females, whereas no difference was found in short-memory ability. Furthermore, aging and gender were found to be significant predictors of ToM ability. The present study is one of few that have been conducted to understand the ToM ability of older people. The findings support the claim that, like some other cognitive abilities, ToM ability is reduced during the aging process. These findings should be replicated by using other tools in order to strengthen the ecological validity of the results.
\end{abstract}

Index Terms - Old age, memory, theory of mind (ToM), gender, cognitive function, reading the mind eyes test, short-term memory, free recall task.

\section{INTRODUCTION}

Old age is characterized by decreased physiological as well as psychological ability [1]. The aim of the present study is to determine whether the theory of mind (ToM) ability of older people as well as their memory abilities decrease compared with the same abilities in a neurotypical younger population.

Aging is generally assessed based on a person's chronological age and functional status [1]. In modern Western societies, old age was defined relative to the retirement age. Thus, the cut-offs were established as 65 and 67 years of age for females and males, [2]. Increased life expectancy and improved health have rendered these ages less relevant as cut-offs in recent years, because people in this age group are healthier, more active, and more educated than in the past; however, they are still labeled by society as old and are therefore discriminated against [1]. Many studies describe the biological as well as social and psychological characteristics of older people. In terms of function, there is

Manuscript received November 29, 2014; revised May 1, 2015.

S. Pearlman-Avnion and T. Muschinsky are with the Deparment of Education, Tel-Hai College, upper Galilee, 12210 Israel (e-mail: shiripearlmanavnion@yahoo.com, tamar.mush@gmail.com).

J. N. Lazar is with is the Deparment of Psychology, Tel-Hai College, Upper Galilee, 12210 Israel (e-mail: lazary@telhai.ac.il). great variety among older adults [3]. Proper nutrition, lifestyle, sport activities, and a calm emotional state are resilience factors for everybody, but they are more critical for older adults and thus influence the aging process [4]. Therefore, the beginning of old age is indefinable [1].

People in late adulthood are thought to continue the patterns of their earlier life; this continuity provides them with a sense of security. Other studies, however, suggest that this age is less stable because aging is characterized by many changes, and people must adapt to a changing environment if they are to maintain their quality of life [5]. Gender differences were found in economic resources as well as in the psychological and social factors that influence functioning and well-being [6].

Declarative and episodic memory abilities decrease over time, whereas nondeclarative memory is not influenced by age. The reduced episodic function is correlated with a decrease in information processing. On the other hand, linguistic knowledge, linguistic wealth, and semantic knowledge remain stable over a person's lifetime. The ability to recognize a known person is preserved, but the ability to remember holistic experience is impaired. The unaware memory and the aware memory abilities are preserved and impaired, respectively [7].

ToM is the ability to understand and interpret human behavior and interactions in different social contexts, and includes the ability to recognize mental states (mind), such as feelings, thoughts, intentions, and desires of the self and surrounding others. This capability, which underlies normal social relationships and connects cognitive abilities and emotions, enables humans to explain and predict the actions and behaviors of others. Thus the ability is very important in social relationships [8]. The first studies of ToM were conducted to determine whether chimpanzees infer states of this kind or not.

People infer ToM to mean the ability to use a system of conceptualizations about states of mind such as the beliefs, thoughts, intentions, and desires of another person [9]-[11]. ToM enables an individual to explain his own behavior and to predict the behaviors of others while relying on mental states [11]. TOM also enables a person to perceive others as separate entities physically and mentally and to understand the inner drives guiding their behaviors [12]. It is well known that ToM is correlated with social abilities, since the ability to understand mental states is associated with nearly every measure of social human behavior. In fact, ToM enables the explanation, prediction, and manipulation of the behavior of others, and no doubt is an important factor in understanding and appropriately responding to social interactions [12]. Moreover, ToM may influence self-development through the understanding of the relationship between the individual 
himself and his understanding of the other's thoughts. Everybody has immediate access to his mental states and he gains access to other's states through the process of reasoning [13].

Many studies support the idea that young children already possess ToM [13]; however, much less is known about ToM beyond early childhood. Following the argument made earlier in this paper, whereas in older adults some cognitive capabilities are impaired, others are preserved. In spite of the loneliness and the change in social functions that individuals experience while aging, some social functions improve during this time. The life experience of older adults as well as maturity may be resilience factors for better social understanding; on the other hand, cognitive deficiency may lead to misunderstanding of social situations [14]. Happé et al. [9] found that people who are 61 to 68 years of age have ToM ability, and that their ability is better than that of people who are 21 to 30 years old. Happé et al. explained their findings in terms of older people's broader experiences of social situations, by which they learn to understand the mental states of others. Maylor et al. [10], on the other hand, found a negative correlation between ToM ability and age. This finding was clearer when ToM ability was mediated by memory ability. The contribution of memory ability to ToM task performance, however, was unclear; therefore, Mayor et al. conducted another study that replicated the experiment conducted by Happé et al. [9], this time using the same stories used by Happé et al., which didn't require the use of memory abilities. The second study yielded a negative correlation between ToM ability and participant age, even when the variable of memory ability was held constant [10].

Thus, there is a debate in the literature. On one hand, Happé et al. [9] argued that ToM ability improved over the life span; on the other, Maylor et al.'s [10] findings implied that ToM ability decreased with age. Sullivan and Ruffman [15] tried to solve this dilemma by attributing the contradictory results to methodological reasons. They conducted another study, which yielded results similar to those of Maylor et al. [10]. But their results were not clear enough to attribute the contrasting results to methodology. Pardini and Nichelli [16] tried to attribute the results to interpersonal differences in mental maturity. They used Baron-Cohen, Wheelwright, Hill, Raste, and Plumb's (2001) Reading the Mind in Eyes test and found reduced ToM ability to be a function of age but also found that the decrease in this ability started already at middle age (55-65 years old) and was not specific to old age [16].

The common public opinion is that, relative to males, females are more sensitive to others, act more emotionally, and understand better the state of mind of others. Empathy refers to one's ability to feel the emotional mental state of another, whereas ToM refers to the cognitive aspect of understanding another's mental state. ToM ability provides evolutionary benefits since the intentions as well as the behaviors of others are usually predictable. Females are thought to be champions in acting properly in social situations as well as in leading in social situations [17]. Nonetheless, only a few studies have been conducted to find the correlation between gender and ToM. The results have been contradictory, and most found little advantage for

\section{females [18].}

The central aim of the present study is to resolve the dilemma occurring in the literature about whether ToM ability is enhanced or reduced as a function of age while controlling for the memory ability variable. A secondary aim of this study is to determine the contribution of gender to ToM ability.

Our first hypothesis was that age would be a significant predictor of ToM ability as well as memory abilities. Our second hypothesis was that females would present better ToM abilities, but not memory abilities, than males. Our third hypothesis was that gender and age would be significant predictors of ToM ability.

\section{METHOD}

\section{A. Participants}

Seventy-three subjects, 37 females and 36 males, participated in the study voluntarily. Their mean age was 65.67 years $(S D=11.03)$, and their ages ranged from 50 to 92 . Twenty-four participants (11 females and 13 males) were between 50 and 60 years old $(M=54.04, S D=2.54)$. Twenty-three participants (12 females and 11 males) were between 60 and 70 years old $(M=63.13, S D=2.62)$. Twenty-six participants (14 females and 11 males) were older than $70(M=78 / 65, S D=5.26)$. All participants spoke Hebrew as their mother language and had normal or corrected-to-normal vision.

\section{B. Apparatus and Stimulus Materials}

Reading the Mind in the Eyes test. To estimate ToM ability, we used the Reading the Mind in the Eyes test (Baron-Cohen et al., 2001). During this test, a participant views 36 images of people, presented one at a time, and cropped to show just the area around the eyes. Each image is surrounded by four emotion-laden words. The participant is asked to choose from the four words the one that best describes the picture. Each correct response is scored one point. The general test score, which ranges from 0 to 36 , is the summation of the participant's correct responses. Test-retest reliability for total score on the Eyes test was .63 $(p<.01)$, based on the intraclass correlation coefficient [19]. Internal consistency (Cronbach's alpha) was .605. Confirmatory factor analysis supported a unidimensional model, with maximal weighted internal consistency reliability equal to .719. Test-retest reliability for the Eyes test, as measured by the intraclass correlation coefficient, was .833 (95\% confidence interval $=.745$ to .902 ). Females scored significantly higher than males on both the Eyes test and the Empathy Quotient (EQ). Those participants who scored lower than 30 on the EQ also scored lower on the Eyes test than those who scored higher than 30 on the EQ $(p<.05)$. Eyes-test scores were not related to social desirability [20].

Memory test. To estimate the participants' memory abilities, we used an immediate free recall task. The task was composed by the researchers of the study and contained 20 words in Hebrew selected to represent six categories: qualities, housewares, professions, foods, animals, and articles of clothing. The list of words was read once to each 
participant slowly (1 word per second). Immediately after the final list item was read, the participant was prompted to recall the items in any order. The total number of correct responses was the score for the task. The average score was 6.11 (SD = 1.86).

\section{Procedure}

Participants completed the tasks individually at their homes. They first took the Reading the Mind in the Eyes test, followed immediately by the free recall test.

\section{RESULTS}

Our first hypothesis was that age would be a significant predictor of ToM ability as well as memory abilities.

Fig. 1 presents the scatter plot of ToM scores as a function of age. As the figure shows, younger participants achieved higher ToM scores compared with older participants. Thus, as predicted, a moderate negative correlation $(r=-.47)$ was found between ToM scores and age. A linear regression model yielded a significant effect $(F(1,71)=20.145, p$ $<.001)$. Age explained $22.09 \%$ of the variance in ToM scores.

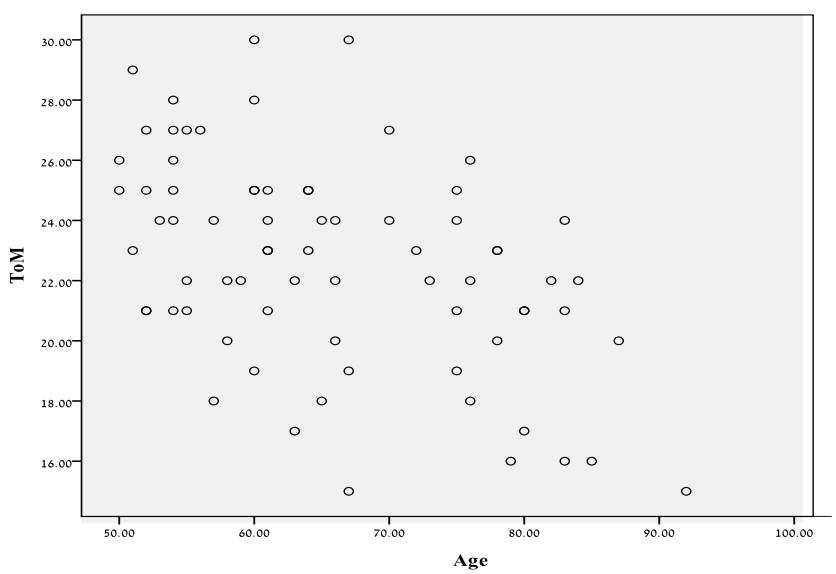

Fig. 1. Theory of mind (ToM) scores as a function of age.

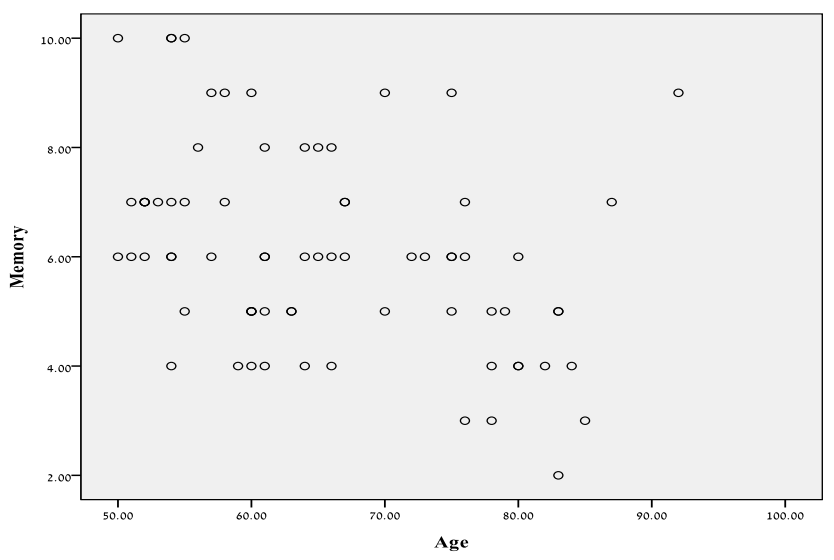

Fig. 2. Memory scores as a function of age.

Fig. 2 presents the scatter plot of memory scores as a function of age. As the figure shows, younger participants achieved higher memory scores compared with older participants. Thus, as predicted, a weak negative correlation $(r=-.382)$ was found between memory scores and age as compared to between ToM scores and age. A linear regression model yielded a significant effect $(F(1,71)=$ $12.120, p=.001)$. Age explained $14.6 \%$ of the variance in the memory scores.
Our second hypothesis was that females would present better ToM abilities, but not memory abilities, than males. As predicted, females' ToM ability was better $(M=23.351, S D$ $=3.276)$ than males' $(M=21.833, S D=3.571)$. A one-way ANOVA of ToM scores revealed a significant effect of gender $[F(1,73)=3.582, p=.05]$. However, females' memory ability was better $(M=6.189, S D=1.883)$ than males' $(M=6.028, S D=1.859)$. A one-way ANOVA of memory scores revealed a nonsignificant effect of gender $(F$ $<1, p>.05)$. In other words, no significant difference was found in short-term memory abilities between females and males.

The third hypothesis was that age and gender would predict ToM ability. Multiple regression analysis with ToM ability as the independent variable and with gender and age as predictors yielded a significant effect $[F(2,72)=12.755, p$ $=.05]$. Gender and age explained $26.7 \%$ in the variance in ToM ability $\left[r^{2}=2.67\right]$. Standard regression coefficients were significant for age $(\beta=-.468)$ and for gender $(\beta=.215)$.

\section{DISCUSSION}

The central aim of the present study was to resolve the dilemma occurring in the literature about whether ToM ability is enhanced or reduced as a function of age while controlling for the memory ability variable. The results of this study show that ToM ability as well as immediate short-term memory ability is significantly reduced as a function of age. Younger people present better ToM and memory abilities compared with older people.

ToM is a congenital ability related to social abilities and to the ability to understand the mental states of others, traits that help a person navigate complicated social situations [21]. Although ToM is a congenital ability, it is stable. ToM ability as well as short-term memory ability was found to be reduced as a function of age due to changes in the neural system and due to environmental factors [22]. Thus our findings resolve the dilemma presented. Furthermore, results for the second and third hypotheses suggest that males' ToM abilities are more sensitive to age influences than are females' ToM abilities.

The results of this study are in line with the selective optimization with compensation theory [23], which suggests the older a person gets, the more aware they become of the age-related benefits and losses they experience [24]. Social as well as cognitive and functional resources are reduced as a function of age; therefore, one should use them carefully, choosing targets that are most applicable and important and investing energy only in those targets. Those targets are chosen at the expense of others, which are of lower priority. As people employ compensation strategies, they rely on closer persons in their surroundings and neglect others. In other words, a general social life is less important and less useful in older age. The outcome is that ToM ability is less adaptive to this period. It may be reduced and, at the same time, other abilities that are more important should be stable.

The socio-emotional selectivity theory (SST) [25] supports our results as well. SST argues that as a function of age, people change the priority of their goals, which become more closely related to emotion and meaning; in turn, they become 
less interested in understanding the state of mind of random others but usually continue to understand their close family members and friends with no effort because they already know them.

Moreover, the resilience-sensitivity theory [26] explains that older adults try to avoid negative experiences. Furthermore, older people who can avoid negative experiences experience higher well-being [26]. Since an emotional relationship with an unknown person might cause pain, they prefer to avoid such a relationship in order to avoid the associated pain. In this case, again, ToM ability is less important in the low-mental-resources condition.

Another interesting finding of this study is the gender difference in ToM ability, which was not found in short-term memory ability. Only a few studies have explored gender differences in ToM ability. The present results are in keeping with Bosacki's [18] findings, which suggest that women are significantly better than men at ToM tasks. Baron-Cohen et al. [27] also found that females are better than males at the Reading the Mind in the Eyes task. Thus the present results replicate and strengthen previous findings using the same and other tasks. The results may be explained by the tendency of women's lifestyles to include more changes [26] and therefore women need to cope better with others. During old age women are more dominant, energetic, and assertive than men, an advantage that helps them to be more open to their social surroundings. In this case ToM ability can be a preference. Short-term memory is less important during this period; thus, despite previous findings [6] that memory ability is weaker in females than in males, the present study suggests no difference. The different findings may be a result of the different memory resources investigated in the studies. Whereas in the present study a free recall task was conducted and represents short-term memory ability, the tasks used in prior studies represent other aspects of memory.

The present study suggests that ToM ability, as well as short-term memory ability, is reduced as a function of age. The ToM ability of older women was found to be better than that of older men, whereas no gender difference was found in short-term memory ability. Finally, age and gender were found to be significant predictors of ToM ability. The results of the present study support those of Slessor et al. [11], who claimed that aging-related structural changes in the frontal and temporal cortex are in line with reduced ToM ability. The present results also support Baron-Cohen et al.'s [27] findings about gender differences. Further studies should be conducted to replicate the results using other tools and to better understand the neuronal basis of the aging process.

\section{REFERENCES}

[1] L. Morgan and S. Kunkel, Aging: The Social Context, Thousand Oaks, CA: Pine Forge, 2001.

[2] T. D. Brothers, O. Theou, and K. Rockwood, "Frailty and migration in middle-aged and older Europeans," Archives of Gerontology and Geriatrics, vol. 58, no. 1, pp. 63-68, 2014.

[3] S. Tucker, R. Baldwin, J. Hughes, S. Benbow, A. Barker, A. Burns, and D. Challis, "Old age mental health services in England: Implementing the national service framework for older people," International Journal of Geriatric Psychiatry, vol. 22, no. 3, pp. 211-217, 2007.

[4] R. Schulz and J. Heckhausen, "A life span model of successful aging," American Psychologist, vol. 51, no. 7, pp. 702-714, 1996.
[5] J. Lynn and D. M. Adamson, Living Well at the End of Life: Adapting Health Care to Serious Chronic Illness in Old Age, Santa Monica, CA: RAND, 2003.

[6] C. Lustig, C. P. May, and L. Hasher, "Working memory span and the role of proactive interference," Journal of Experimental Psychology: General, vol. 130, no. 2, pp. 199-297, 2001.

[7] W. Hoyer and P. A. Roodin, "Memory, attention, and learning," in Adult Development and Aging, $6^{\text {th }}$ ed. pp. 176-208, Boston, MA: McGraw-Hill, 2009.

[8] D. Premack and G. Woodruff, "Does the chimpanzee have a theory of mind?" Behavioral and Brain Sciences, vol. 1, no. 4, pp. 515-526, 1978.

[9] F. Happé, E. Winner, and H. Brownell, "The getting of wisdom: Theory of mind in old age," Developmental Psychology, vol. 34, no. 2, pp. 358-362, 1998.

[10] E. Maylor, J. Moulson, A. Muncer, and L. Taylor, "Does performance on theory of mind tasks decline in old age?" British Journal of Psychology, vol. 93, pp. 465-485, 2002.

[11] G. Slessor, L. Phillips, and R. Bull, "Exploring the specificity of age-related differences in theory of mind tasks," Psychology and Aging, vol. 22, no. 3, pp. 639-643, 2007.

[12] T. Paal and T. Bereczkei, "Adult theory of mind, cooperation, Machiavellianism: The effect of mindreading on social relations," Personality and Individual Differences, vol. 43, no. 3, pp. 541-551, 2007.

[13] B. Sodian, C. Hulsken, and C. Thoermer, "The self and action in theory of mind research," Consciousness and Cognitions, vol. 12, pp. 777-782, 2003.

[14] N. R. Hooyman and H. A. Kiyak, "Social theories of aging," in Social Gerontology: A Multidisciplinary Perspective, $8^{\text {th }}$ ed. Boston, MA: Pearson, 2009, pp. 305-332.

[15] S. Sullivan and T. Ruffman, "Social understanding: How does it fare with advancing years?" British Journal of Psychology, vol. 95, pp. 1-18, 2004.

[16] M. Pardini and P. F. Nichelli, “Age-related decline in mentalizing skills across adult life span," Experimental Aging Research, vol. 35, pp. 98-106, 2009

[17] S. Krach, I. Blumel, D. Marjoram, T. Lataster, L. Krabbendam, J. Weber, and T. Kircher, "Are women better mindreaders? Sex differences in neural correlates of mentalizing detected with functional MRI," BMC Neuroscience, vol. 10, no. 9, pp. 1-11, 2009.

[18] S. L. Bosacki, "Theory of mind and self-concept in preadolescents Links with gender and language," Journal of Educational Psychology, vol. 92 , no. 4, pp. 709-717, 2000.

[19] E. G. Fernández-Abascal, R. Cabello, P. Fernández-Berrocal, and S. Baron-Cohen, "Test-retest reliability of the "Reading the Mind in the Eyes' test: A one-year follow-up study," Molecular Autism, vol. 4, no. $1,2013$.

[20] M. Vellante, S. Baron-Cohen, M. Melis, M. Marrone, D. R. Petretto, C. Masala, and A. Preti, "The 'Reading the Mind in the Eyes' test: Systematic review of psychometric properties and a validation study in Italy," Cognitive Neuropsychiatry, vol. 18, no. 4, pp. 326-354, 2013

[21] M. Siegal and R. Valey, "Neural systems involved in 'theory of mind'," Neuroscience, vol. 3, pp. 467-461, 2002.

[22] S. T. Charles and L. L. Carstensen, "Social and emotional aging," Annual Review of Psychology, vol. 61, pp. 383-469, 2009.

[23] P. B. Baltes and M. M. Baltes, "Psychological perspectives on successful aging: The model of selective optimization with compensation," Successful Aging: Perspectives from the Behavioral Sciences, vol. 1, pp. 1-34, 1990.

[24] M. Marsiske, F. B. Lang, P. B. Baltes, and M. M. Baltes, "Selective optimization with compensation: Life-span perspectives on successful human development," in R. A. Dixon and L. Backman, Eds Compensation for Psychological Deficits and Declines: Managing losses and Promoting Gains, pp. 35-79, Hillsdale, NJ: Erlbaum, 1995.

[25] L. L. Carstensen, "The influence of a sense of time on human development," Science, vol. 312, no. 5782, pp. 1913-1915, 2006.

[26] S. T. Charles and J. R. Piazza, "Strength and vulnerability across the lifespan: An integration of literature on aging, emotional well-being and emotion regulation," Social and Personality Psychology Compass, 2009.

[27] S. Baron-Cohen, S. Wheelwright, J. Hill, Y. Raste, and I. Plumb, The "Reading the Mind in the Eyes," Test Revised Version: A study with normal adults, and adults with Asperger syndrome or high-functioning autism, Journal of Child Psychology and Psychiatry, vol. 42, no. 2, pp. 241-251, 2001. 


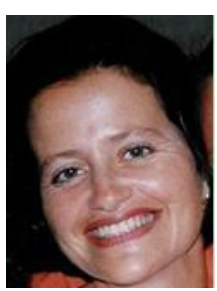

Shiri Pearlman-Avinion was born in Israel. She received her B.A. is in psychology and art history from Department of Psychology, Haifa, Israel. She received her M.A. in cognitive psychology from Department of Psychology in Haifa University. She received her Ph.D from Haifa University in August 2002. Her PhD paper title is sociability and language use among people with development disabilities: Social and informative aspects of theory of mind. Now she is a lecturer at the Department of Education, in Tel-Hai College, Upper Galilee, Israel. Parallel to researching, she is also a school psychologist and owns a clinic.

Dr. Shiri Pearlman-Avinion's interests in research fields are autism, cognition, theory of mind, and educational psychology.

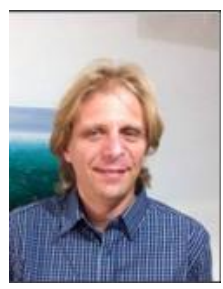

Josef N. Lazar was born in Haifa, Israel. He studied behavioral cognitive neuroscience at Tel-Aviv University, Israel in the framework of Direct $\mathrm{PhD}$ program for excellent student. He received his B.A. degree in psychology from the Open University, Israel. $\mathrm{He}$ received his B.Sc. degree in biology fromthe Technion, Haifa, Israel. He is a school psychologist at the municipal school psychology service and owns a clinic. $\mathrm{He}$ is a member of the ethics forum of educational psychologists.

Dr. Josef N. Lazar's interests in research fields are educational psychology, neuroscience, affect, cognition, theory of mind, memory, and gender differences. Some of his books are "Affect and attention" and "Selective Mutism: An-Evidenced Based Integrative Treatment Model".

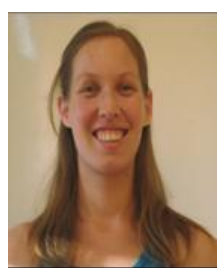

Tamar Muschinsky was born in 1985, in Kfar Mordechai. She completed her high school education at 'Tsafit' High school in Kibbutz Kfar Menachem. She received her B.A. in education studies from Tel-Hai College, Upper Gallee, Israel. She received her M.A. degree in diagnosis and treatment of learning disabilities, at the Hebrew University, Jerusalem in 2014. She has been working in "Otiyot"

institute in Jerusalem.

Ms. Tamar Muschinsky is interested in the field of remedial education and the effect it has on children, adolescents and adults. Particularly, she wants to explore whether remedial education has an affect on learning difficulties adults encounter on a daily bases at work, or when they want to study, when they haven't been treated in small age. 